\title{
O buraco negro e sua sombra
}

The black hole and its shadow

Antes a sombra do que a luz? Será possível?

(Friedrich Nietzsche, O Andarilho e sua Sombra)

\author{
Juliano C. S. Neves ${ }^{*}[0$ \\ ${ }^{1}$ Universidade Federal do ABC, Centro de Ciências Naturais e Humanas, Santo André, SP, Brasil.
}

Recebido em 22 de maio de 2020. Revisado em 12 de agosto de 2020. Aceito em 16 de setembro de 2020.

\begin{abstract}
A mais comentada imagem científica em 2019 foi a imagem de M87*, buraco negro supermassivo central da galáxia Messier 87. A partir da observação da colaboração Event Horizon Telescope, fomos capazes de ver um buraco negro. Na verdade, a famosa imagem mostra-nos a sombra de M87*. Neste artigo, será introduzido o conceito de sombra de um buraco negro e indicada a sua importância para pesquisas nas áreas de gravitação e cosmologia.
\end{abstract}

Palavras-chave: Buraco Negro; Sombra; Relatividade Geral.

The most commented scientific image in 2019 was the image of M87*, the central supermassive black hole at the Messier 87 galaxy. From the Event Horizon Telescope's observation, we were able to see a black hole. Indeed, the famous image shows us the shadow of M87*. In this article, the concept of black hole shadow will be introduced as well as its importance to researches in gravitation and cosmology.

Keywords: Black Hole; Shadow; General Relativity.

\section{Introdução}

A colaboração internacional Event Horizon Telescope (EHT) produziu uma das mais comentadas imagens na ciência em décadas 1, 2]. A imagem do buraco negro central na galáxia Messier 87, indicado por M87*, foi noticiada por diversos meios. De certa forma, a imagem de M87* é a imagem do fim do mundo. Pois, como sabemos, a partir da teoria da relatividade geral, um corpo com massa não pode escapar de um buraco negro, nem mesmo a luz escapa do intenso campo gravitacional de um buraco negro 11 Portanto, um buraco negro, como o M87*, poderia ter um portal com as mesmas inscrições do inferno de Dante: "Deixai toda esperança, ó vós que entrais.' 2 2 limite entre aquilo que está dentro e aquilo que está fora é demarcado pelo horizonte de eventos. Quando se cruza um horizonte de eventos, não se escapa mais de um buraco negro. No entanto, ao contrário do que foi anunciado em alguns canais e mídias sociais, a imagem de $\mathrm{M}^{7 *} 7^{*}$ não desvela um horizonte de eventos. É a sombra do buraco negro supermassivo M87* aquilo que vemos, uma região escura que indica a ausência de estrelas e galáxias ao fundo.

\footnotetext{
*Endereço de correspondência: nevesjcs@if.usp.br

${ }^{1}$ Se pensarmos apenas a partir da relatividade geral, um buraco negro será uma prisão perfeita. No entanto, se à teoria einsteiniana adicionarmos a teoria quântica de campos, um buraco negro poderá, então, emitir algo. E esse algo é chamado radiação Hawking. Veja Matsas [3] para uma introdução ao tema na Revista Brasileira de Ensino de Física.

${ }^{2}$ Alighieri 4], canto III, verso IX.
}

A colaboração EHT realizou um grande trabalho para compor a primeira imagem de um buraco negro. Foram usadas oito estações com radiotelescópios espalhados pela América, Antártida, Europa e Havaí. Por meio da interferometria de longa linha de base (very-long-baseline interferomety, VLBI), que sincronizou as estações com seus radiotelescópios, a técnica explorou a rotação do planeta Terra para formar um grande radiotelescópio (com a associação das oito estações) e realizar observações no comprimento de onda de $1.3 \mathrm{~mm}$. A potência da técnica empregada era exaltada antes mesmo da observação de M87*. Pois a colaboração internacional sabia da enorme capacidade que tinha disponível, uma resolução angular de 20 micro arc-segundos. Tal resolução torna a capacidade de amplificação do enorme radiotelescópio incrível, apta a permitir a leitura de um jornal, disposto num café em Paris, por alguém sentado num agradável banco no belo Central Park em Nova Iorque. Assim, após décadas de desenvolvimento da técnica de interferometria e de observações, além de muito processamento de dados, a imagem do longínquo M87* foi composta.

Portanto, a partir dos dados ou da imagem, qual é o melhor candidato para M87*? Certamente, um buraco negro. Mas entre os buracos negros ou entre as soluções das equações do campo gravitacional de Albert Einstein 5], qual é a mais apropriada para a imagem vista por nós? A colaboração internacional não hesitou em afirmar que a imagem de M87* confirma a teoria einsteiniana assim como o buraco negro de Kerr (ou geometria, ou espaço- 
tempo de Kerr) é um candidato adequado à descrição da sombra de M87*.

O buraco negro de Kerr foi desenvolvido, como uma solução das equações de Einstein, em 1963 por Roy Kerr [6]. Trata-se de um corpo com massa bem determinada e neutro eletricamente. Tem rotação sobre o seu eixo e uma estrutura espaço-temporal muito mais complicada do que o estático buraco negro de Karl Schwarzschild [7], considerado a primeira solução das equações de Einstein proposta por alguém que não o próprio Einstein ${ }^{3} \mathrm{Na}$ Seção III, olharemos com mais atenção à geometria de Kerr e veremos quais sombras produz.

Os primeiros trabalhos sobre sombras de buracos negros datam da segunda metade do século XX. John Synge foi pioneiro quando determinou a sombra do buraco negro de Schwarzschild [9]. Já James Bardeen, em seguida, fez o cálculo para o buraco negro de Kerr 10]. Décadas depois, sombras para diversos buracos negros no contexto einsteiniano [11 17] e em contextos além da teoria de Einstein 18 20 foram e estão sendo calculadas. Uma sombra é determinada por órbitas instáveis que estão fora do horizonte de eventos. Na região que forma a silhueta da sombra, fótons em tais órbitas instáveis podem cair na direção do buraco negro ou podem escapar para serem detectados pelos radiotelescópios da EHT. Para um buraco negro como o de Schwarzschild, um que não tem rotação, a sombra é perfeitamente circular, e as órbitas instáveis que determinam a silhueta da sombra ficam a $r_{f}=3 M$ do centro do buraco negro (ou $3 G M / c^{2}$ no S.I., sendo que $G$ é a constante gravitacional, $M$ é a massa do buraco negro, e $c$ é a velocidade da luz no vácuo). Não esqueçamos que o raio de Schwarzschild ou o raio do horizonte de eventos para esse buraco negro estático é $2 M$ (ou $2 G M / c^{2}$ no S.I.), evidenciando assim que o fenômeno sombra é exterior ao horizonte de eventos. Já para um buraco negro com rotação, sua sombra nem sempre é circular, pode ser achatada. Como veremos, tal efeito é produzido pela alta rotação do buraco negro (em torno do seu eixo) e pelo ângulo em que a sombra é observada.

O artigo organiza-se de tal modo: na Seção 2 o fenômeno sombra será definido; na Seção 3 será apresentada e discutida a geometria ou buraco negro girante de Kerr, além de serem mostradas as equações das geodésicas para esse espaço-tempo. Por fim, um procedimento para construção de sombras será indicado. Na Seção 4, aplicações das sombras em algumas áreas e campos de pesquisa serão listadas. Os comentários finais encontram-se na Seção 5 Utilizo, para fins de simplicidade nas equações apresentadas, as unidades geométricas, segundo as quais a constante da gravitação e a velocidade da luz no vácuo são iguais à unidade, i.e., $G=c=1$.

${ }^{3}$ Para uma leitura histórica dessa importante solução ou conquista da teoria einsteiniana, veja Saa [8] sobre os 100 anos da solução de Schwarzschild.

\section{O Fenômeno Sombra}

De certa forma, o fenômeno sombra era, mesmo antes da colaboração EHT, algo já conhecido. O primeiro teste da teoria da relatividade geral foi propiciado pelo desvio da luz provocado pelo campo gravitacional do Sol.4 Uma sombra é igualmente um fruto do desvio da luz por um intenso campo gravitacional. Mas ao contrário do desvio da luz medido no eclipse de 1919 em Sobral, no Ceará, o primeiro bem-sucedido teste da teoria da relatividade geral, em que o Sol foi descrito aproximadamente como uma massa sem rotação, para sombras é necessário o uso de uma geometria que descreva uma massa com rotação, como é o caso da métrica ou geometria de Kerr. Uma sombra de um buraco negro em rotação, como o M87*, apresenta-se levemente deformada. Como a colaboração relatou, no caso da sombra de M87*, há um desvio da circularidade menor do que $10 \%$ para a sua sombra. Portanto, sua sombra é quase um circulo perfeito. Um desvio da circularidade de uma sombra somente é possível para um buraco negro em rotação. É esse o motivo para, na Sec. 3. darmos uma olhada na métrica, geometria ou buraco negro de Kerr com o intuito de interpretar o fenômeno sombra a partir da imagem levemente deformada (veja figura 1) e dos parâmetros divulgados pela EHT.

O fenômeno sombra é a obstrução de uma região do céu estrelado por um buraco negro. Se pensarmos que observamos estrelas e galáxias ao fundo, com um buraco

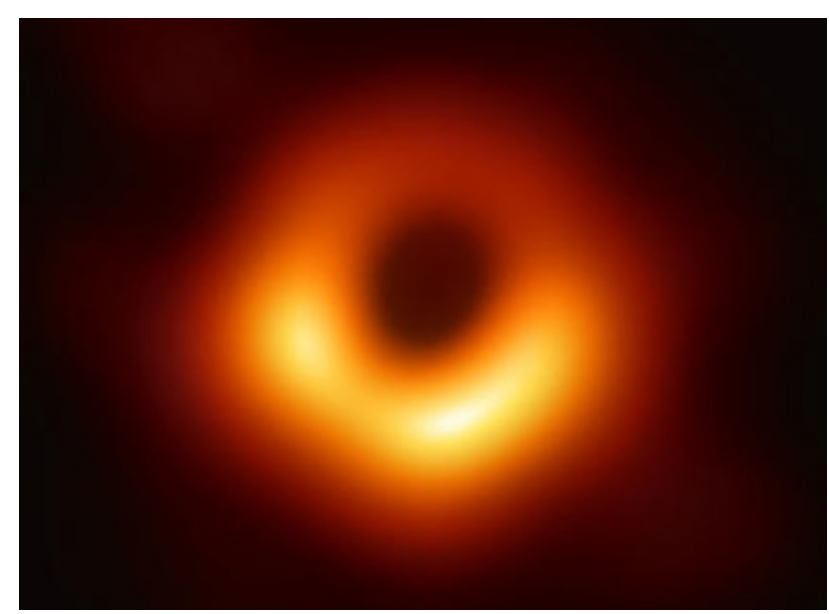

Figura 1: A amplamente divulgada imagem da sombra do buraco negro M87* no centro da galáxia Messier 87, construída por interferometria pela colaboração internacional Event Horizon Telescope [1] (imagem disponível no site https://eventhorizontelescope.org/press-release-april-102019-astronomers-capture-first-image-black-hole). A sombra é a área escura, envolvida pela região avermelhada-alaranjada que indica a matéria que orbita e cai no buraco negro. O limite da região interna escura é a silhueta da sombra.

${ }^{4}$ Para uma leitura sobre o primeiro teste da relatividade geral em
Sobral, a Revista Brasileira de Ensino de Fúsica publicou um
número especial em comemoração aos seus 100 anos. Veja, por número especial em comemor
exemplo, os artigos $21,23$. 
negro entre o fundo estrelado e nós, haverá uma região sem brilho, escura, cuja silhueta marca o limite da sombra do buraco negro. Como podemos ver na figura 2 (em cima), raios de luz de estrelas atrás do buraco negro (em relação à nossa posição ou a de um observador) podem cair no buraco negro ou atingir o nosso observador. Haverá trajetórias da luz que passam na borda da sombra. Tais trajetórias delimitam a chamada silhueta da sombra. São órbitas instáveis, podem conduzir ao buraco negro ou ao seu exterior. Portanto, em $r=r_{f}$ temos uma região de fótons aprisionados ou desviados pelo campo gravitacional do buraco negro, uma região externa ao horizonte de eventos chamada de esfera de fótons. Se assumirmos que o raio do horizonte de eventos é $r=r_{h}$, $\log \mathrm{O}$

$$
r_{f}>r_{h}
$$

No entanto, como podemos ver na figura 2 (em baixo), devido ao desvio da luz promovido pela grande massa de um buraco negro, efeito também chamado de lente gravitacional, o observador e os radiotelescópios da EHT medem um raio ou diâmetro para a sombra maior do que o raio ou diâmetro da esfera de fótons. Como foi mencionado
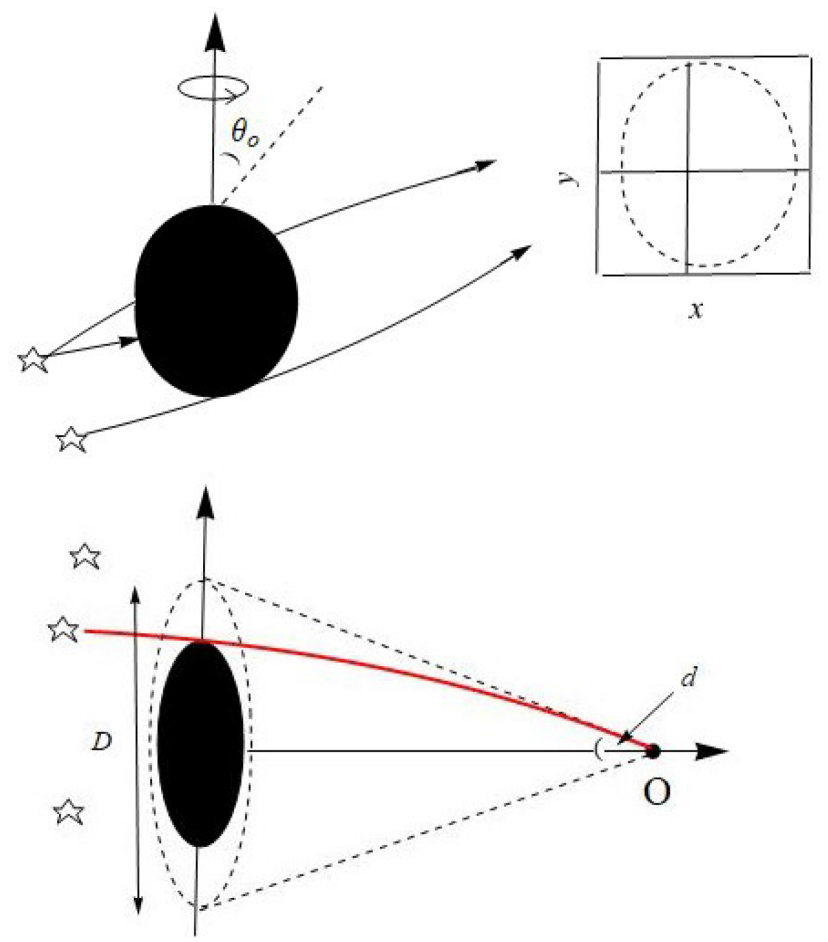

Figura 2: (Em cima) Na representação, a sombra está entre o fundo estrelado e o observador que a mapeia com o uso das coordenadas $x$ e $y$. O distante observador coloca-se a um ângulo $\theta_{o}$ em relação ao eixo de rotação do buraco negro. (Em baixo) Nosso observador O observa um diâmetro aparente $D$ para a sombra maior do que o diâmetro da esfera de fótons (região escura que captura os raios de luz). Devido ao efeito do desvio dos raios de luz (deflexão da luz) pela grande massa do buraco negro, o observador constatará, a partir do diâmetro angular do fenômeno $\left(d=42 \pm 3 \mu\right.$ as para $\left.\mathrm{M} 87^{*}\right)$, um diâmetro linear para sombra maior do que o diâmetro da esfera de fótons. anteriormente, para o buraco negro de Schwarzschild $r_{f}=3 M$, já o raio de sua sombra, devido ao efeito gravitacional, é de $3 \sqrt{3} M$. Para obter as trajetórias para as quais $r=r_{f}$, precisamos das equações das geodésicas para o buraco negro em questão. E como, neste artigo, o buraco negro escolhido é o de Kerr, veremos então as equações das geodésicas para essa geometria.

O distante observador, segundo a figura 2 (em cima), localizado em $r=r_{o b s} \gg r_{f}$, observa a sombra e usa coordenadas especiais para mapeá-la. Coordenadas celestes são usadas para descrever a sombra. Quando tais coordenadas são projetadas num plano cartesiano, temos então um círculo ou um quase círculo que representa a sombra ou a sua silhueta.

Como podemos notar na figura 1, na imagem divulgada pela EHT, há no entorno da sombra uma região avermelhada-alaranjada. Nessa região existe matéria (gás e poeira) orbitando e caindo em direção ao M87*. Nós a enxergamos porque em seu movimento essa matéria emite, por exemplo, raios X. Portanto, deve-se esperar que uma sombra não esteja a sós, um disco de acreção com matéria ionizada ou plasma é concebido no entorno de uma sombra. Há um campo de estudo atualmente que diz respeito à melhor maneira de modelar a vizinhança de um buraco negro, seja usando plasma ou matéria escura. É um campo fértil dentro da atual física de buracos negros 5 Neste artigo, nas sombras construídas a partir da geometria de Kerr, considerarei, por simplicidade, um buraco negro girante no completo vácuo. Tal simplificação pode ser justificada quando a matéria avermelhada-alaranjada não tem uma massa incrivelmente grande, podendo assim ser ignorada a sua influência na geometria ou nas formas do espaço-tempo e da sombra em estudo.

Toda sombra é observada a partir de um ângulo, indicado aqui por $\theta_{o}$, que é o ângulo em que um observador mapeia a sombra, o seu ângulo a partir do eixo de rotação do buraco negro (veja figura 2). O valor de $\theta_{o}$ na observação famosa é estimado pela colaboração a patir de jatos emitidos pelo buraco negro supermassivo M87*. Como é suposto que tais jatos são emitidos na direção do eixo de rotação de um buraco negro, a observação dos jatos de M87* indica $17^{\circ}$. Portanto, assume-se que o buraco negro está sendo observado àquele ângulo. Outro parâmetro que diz respeito à observação - e não a uma característica em si do objeto - é a nossa distância em relação ao M87*. A colaboração EHT relata o valor $r_{o b s}=(16.8 \pm 0.8) \mathrm{Mpc}$ (em megaparsec ou, aproximadamente, 55 milhões de anos-luz de distância). O diâmetro angular do objeto observado (incluindo a área avermelhada-alaranjada) é outro parâmetro observacional que diz respeito à nossa posição em relação ao fenômeno observado. Seu valor obtido pela EHT é de $d=42 \pm 3 \mu$ as (em micro arco-segundo). Por fim, a sombra de M87* é levemente deformada, seu desvio da circularidade é menor do que $10 \%$, ou seja, $\Delta C \lesssim 0.1$, e isso indica que $\mathrm{M} 87^{*}$ não é estático, mas gira. Como

\footnotetext{
${ }^{5}$ Veja Cunha e Herdeiro [24] para uma revisão do tema.
} 
disse, um desvio da circularidade diferente de zero só é possível a um objeto com rotação sobre o seu próprio eixo.

Outros parâmetros importantes de M87* são a sua massa e o seu momento angular. E tais parâmetros são intrínsecos ao objeto observado, não dependem da posição relativa do observador. Sua massa é bem estabelecida pela EHT, é $M=(6.5 \pm 0.7) \times 10^{9} M_{\odot}$, onde $M_{\odot}$ é a massa do Sol. Já o seu momento angular ainda é um parâmetro que está sob debate. Estimativas feitas a partir da deformação da sombra ou do seu desvio da circularidade rejeitam um valor muito pequeno para a rotação do buraco negro. Tal deformação, para o ângulo de $17^{\circ}$ em que é observado, só é possível para um buraco negro que gira apressadamente. Na próxima seção, veremos quão rápido pode ser esse movimento.

\section{Um Buraco Negro com Rotação}

\subsection{A geometria}

$\mathrm{Na}$ teoria da relatividade geral, como disse, a métrica ou geometria de Kerr descreve um corpo em rotação. Se a geometria de um corpo estático foi construída por Schwarzschild logo após Einstein divulgar as suas equações do campo gravitacional em 1916, foram necessários quase 50 anos para que alguém construísse um espaçotempo que descreve um corpo em rotação no contexto einsteiniano. Roy Kerr o fez num já clássico trabalho 6] . $\mathrm{Na}$ forma mais adotada, nas chamadas coordenadas de Boyer-Lindquist ${ }^{6}(t, r, \theta, \phi)$, a métrica de Kerr é escrita como

$$
\begin{aligned}
d s^{2}= & g_{\mu \nu} d x^{\mu} d x^{\nu}=-\frac{1}{\Sigma}\left(\Delta-a^{2} \operatorname{sen}^{2} \theta\right) d t^{2}+\frac{\Sigma}{\Delta} d r^{2} \\
& +\Sigma d \theta^{2}+\frac{\operatorname{sen}^{2} \theta}{\Sigma}\left[\left(r^{2}+a^{2}\right)^{2}-\Delta a^{2} \operatorname{sen}^{2} \theta\right] d \phi^{2} \\
& -\frac{4 a M r}{\Sigma} \operatorname{sen}^{2} \theta d t d \phi,
\end{aligned}
$$

sendo que as funções da métrica $\Delta$ e $\Sigma$ são definidas como

$$
\Delta=r^{2}+a^{2}-2 M r \quad \text { e } \quad \Sigma=r^{2}+a^{2} \cos ^{2} \theta .
$$

Aqui, as coordenadas $r, \theta$ e $\phi$ têm o mesmo significado das coordenadas de um problema tratado com coordenadas esféricas, sendo $r$ a coordenada radial, $\theta$ o ângulo polar, e $\phi$ é o ângulo azimutal. E, claro, $t$ é a coordenada temporal na geometria em destaque. Os parâmetros $a$ e $M$ na métrica (2) são o parâmetro de rotação, que se relaciona com o momento angular $J$ do corpo por $a=J / M$, e a massa do buraco negro, respectivamente. Como podemos notar diretamente da equação (2), a métrica de Kerr não é diagonal, quando escrita como uma matriz $g_{\mu \nu}$. Há elementos fora da diagonal principal, são eles $g_{t \phi}=g_{\phi t}$, ao contrário da geometria de Schwarzschild que pode ser

${ }^{6}$ Veja Boyer e Lindquist [25]. escrita como uma matriz diagonal. Isso torna cálculos, como o de geodésicas, a partir da métrica de Kerr, algo muito mais complicado.

A geometria ou espaço-tempo de Kerr é uma solução exata das equações de Einstein

$$
G_{\mu \nu}=8 \pi T_{\mu \nu},
$$

em que $G_{\mu \nu}$ é o tensor de Einstein (diz respeito à geometria), e $T_{\mu \nu}$ é o tensor energia-momento (diz respeito à matéria e à energia do espaço-tempo). A geometria de Kerr é uma solução com simetria axial, ao contrário da de Schwarzschild que tem um grau maior de simetria por possuir a simetria esférica. Ou seja, a geometria de Kerr é simétrica em relação ao eixo segundo o qual um corpo ou um buraco negro com massa $M$ roda. Como roda, têm momento angular $J$. Fazendo-se $a=0$, o momento angular é nulo, logo temos a métrica de Schwarzschild ou um corpo sem rotação. Em Kerr, o corpo gira no vácuo, portanto o tensor energia-momento é nulo, i.e., $T_{\mu \nu}=0$. A métrica (2) possui dois dos três possíveis parâmetros que definem qualquer buraco negro: são eles a massa, o spin ou o momento angular e a carga (podendo ser elétrica e, até mesmo, uma ainda não observada carga magnética). À Kerr só lhe falta a carga 7 É o teorema no-hair (ou teorema da calvície) aquele que afirma que um buraco negro - descrito pela teoria da relatividade geral acoplada ao electromagnetismo - é caracterizado por apenas três parâmetros 8

A função $\Delta$ da métrica tem grande importância. Seus zeros ou raízes indicam os raios do horizonte de eventos $r=r_{h}$ e do horizonte interno $r=r_{i}$. Ao contrário do buraco negro de Schwarzschild, em Kerr há um segundo horizonte, um interno, que para o fenômeno sombra não tem relevância. Os zeros de $\Delta$ indicam

$$
r_{h}=M+\sqrt{M^{2}-a^{2}} \quad \text { e } \quad r_{i}=M-\sqrt{M^{2}-a^{2}},
$$

e podemos ver que quando $a=0$, temos $r_{h}=2 M$, que é o raio de Schwarzschild. A condição $a^{2} \leq M^{2}$ na métrica de Kerr diz respeito à existência de horizontes, pois caso essa desigualdade seja violada não há raízes reais para $\Delta$, ou seja, $r_{i}$ e $r_{h}$ tonam-se números complexos. Tal condição é um limite superior à rotação do buraco negro. Caso não seja obedecida, o buraco negro de Kerr é substituído por uma singularidade nua, uma singularidade que não é escondida ou "vestida" por um horizonte de eventos ${ }^{9}$

${ }^{7}$ A solução das equações de Einstein para um corpo ou buraco negro com rotação e carga é a solução de Kerr-Newman.

${ }^{8}$ Veja Carroll 26], capítulo 6, para uma discussão sobre o tema. Aqui calvície significa que caso um buraco negro "engula" matéria em diversas configurações, o resultado disso será um buraco negro descrito por apenas três parâmetros, e toda a informação e característica daquilo que entrou é definitivamente perdida, como os fios de cabelo de um potencial careca.

${ }^{9}$ Claro, desde que aceitemos a noção de singularidade como algo "real". No entanto, buracos negros regulares não têm uma singularidade e são possíveis mesmo na teoria da relatividade geral (veja a referência 27] para uma apresentação do tema na Revista Brasileira de Ensino de Fúsica). Um estudo sobre a ficcionalidade do conceito problemático de singularidade é encontrado em Neves 28. 
Além dos horizontes, a métrica (2) indica uma região especial na geometria de Kerr. É a ergoesfera, algo exterior ao horizonte de eventos, uma região em que não há observadores estacionários. Em tal região, um observador é arrastado pela rotação do buraco negro, é o fenômeno do arrasto do espaço-tempo que ocorre na ergoesfera, fenômeno que, como veremos, influencia a forma da sombra. Na linguagem técnica da geometria diferencial, o limite da ergosfera, a chamada superfície do limite estacionário, indicada por $S_{+}$na figura 3 dá-se por uma superfície de Killing em que vetores de Killing tangentes a tal superfície são nulos, i.e., têm norma igual a zero (vetores de Killing proveem quantidades conservadas, como veremos). Tal como para os horizontes, em Kerr existe igualmente uma superfície interna do limite estacionário, indicada por $S_{-}$. As superfícies do limite estacionário, que encerram regiões proibitivas a um observador estacionário, são dadas por zeros de outra função, nesse caso são zeros da função métrica $g_{t t}$. Suas expressões são

$$
S_{ \pm}=M \pm \sqrt{M^{2}-a^{2} \cos ^{2} \theta}
$$

coincidindo com os raios dos horizontes interno e externo nos polos, quando $\theta=0$ (veja figura 3). A ergosfera, como uma região especial no espaço-tempo de Kerr, localiza-se então entre $r_{h}$ e $S_{+}$, é a região em que a coordenada radial assume os valores $r_{h}<r<S_{+}$. Observadores estacionários somente são possíveis para além da superfície do limite estacionário, fora de ergosfera, i.e., para $r>S_{+}$.

O conceito de ergosfera, por sua característica peculiar, possibilitou uma engenhosa construção de Roger Penrose [29]. O mecanismo de Penrose é uma forma de "roubar"

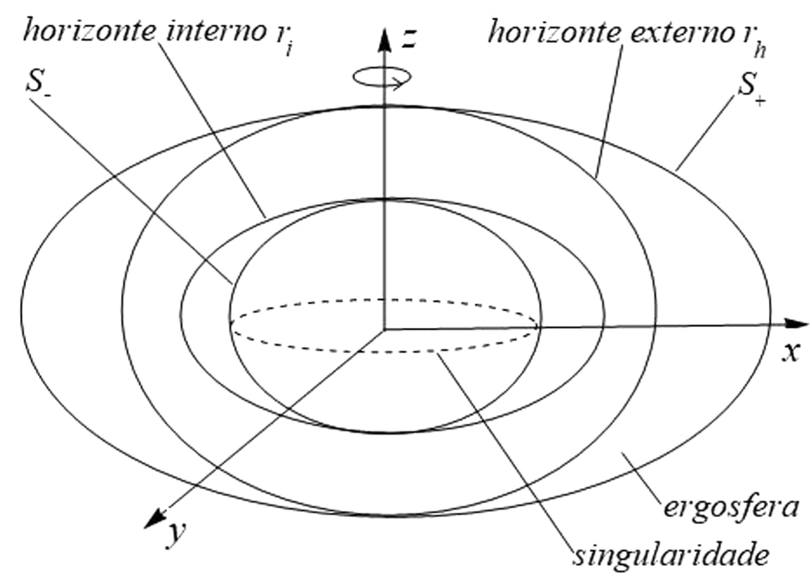

Figura 3: A estrutura do espaço-tempo de Kerr e a indicação das quatro superfícies especiais. A superfície cujo raio é $r_{i}$ indica o horizonte interno, já $r_{h}$ é o raio do horizonte externo, que é o próprio horizonte de eventos para quem está fora do buraco negro. As superfícies $S_{-}$e $S_{+}$são as superfícies interna e externa do limite estacionário, respectivamente. A ergosfera não é uma superfície mas uma região entre $r_{h}$ e $S_{+}$. Por fim, a linha tracejada no centro é a singularidade na geometria de Kerr. A singularidade neste buraco negro é "anelar". energia de um buraco negro em rotação. Extrai-se a energia do buraco negro, diminuindo a sua rotação. Tal mecanismo só é possível em buracos negros que possuem uma ergosfera. 10

Por fim, um comentário sobre os extremos da geometria ou buraco negro de Kerr. Para valores grandes da coordenada radial $r$, o espaço-tempo de Kerr tende ao espaço-tempo de Hermann Minkowski. Ou seja, a métrica de Kerr é assintoticamente plana, seu limite, quando $r \rightarrow \infty$, é o espaço-tempo de Minkowski. Por outro lado, quando olhamos para dentro do buraco negro de Kerr, encontramos uma singularidade que, ao contrário da singularidade de Schwarzschild, não é um ponto. A singularidade em Kerr tem a "forma" de um anel (veja figura 3). Uma singularidade, seja a de Schwarzschild ou a de Kerr, diz respeito à infinitude de quantidades físicas e geométricas ou, mais precisamente, à descontinuidade de geodésicas 11

\subsection{As geodésicas}

Foi Brandon Carter [30] quem calculou, pela primeira vez, as geodésicas da geometria de Kerr. Como a massa de um corpo (seja em repouso ou em movimento) altera a geometria ou a "forma" do espaço-tempo, para cada solução das equações de Einstein que especifica uma dada condição a um corpo ou a vários corpos, temos então geodésicas diferentes. Portanto, geodésicas no espaçotempo de Schwarzschild são diferentes das do espaçotempo de Kerr. Neste, como vimos, há a rotação como um parâmetro a mais para o buraco negro.

Geodésicas são curvas especiais na teoria da relatividade geral. Numa geodésica $\gamma(\tau)$, parametrizada pelo parâmetro $\tau$, o vetor tangente $v^{\mu}=d x^{\mu} / d \tau$ em cada ponto da curva é transportado paralelamente a si mesmo. 12 Como bem sabemos, no espaço plano geodésicas são linhas retas. Mas em geral, na geometria não plana, na geometria riemanniana, que é a área da matemática que propiciou a teoria da relatividade geral, geodésicas obedecem à seguinte equação:

$$
\frac{d^{2} x^{\mu}}{d \tau^{2}}+\Gamma_{\nu \gamma}^{\mu} \frac{d x^{\nu}}{d \tau} \frac{d x^{\gamma}}{d \tau}=0
$$

Há três tipos de vetores na relatividade geral: tipo tempo, tipo luz e tipo espaço. Diferenciam-se entre si pelo valor que suas normas assumem. Na convenção usada aqui, um vetor é tipo tempo se $v^{2}=v^{\mu} v_{\mu}<0$, tipo luz se $v^{2}=0$ e tipo espaço caso $v^{2}>0$. Como para o fenômeno sombra devemos nos ater às geodésicas tipo luz, que são as trajetórias de raios de luz, tais geodésicas têm vetores tangentes - propagados paralelamente a si mesmos - com norma nula. A equação (7) contém

\footnotetext{
${ }^{10}$ Veja Carroll [26], capítulo 6 , para uma introdução ao estudo sobre a ergosfera e o mecanismo de Penrose.

${ }^{11}$ Em Wald 31, capítulo 9, há um tratamento detalhado sobre singularidades na relatividade geral.

12 Para uma compreensão mais precisa do conceito de geodésica, veja Carroll 26], capítulo 3, e Wald 31], igualmente o capítulo 3.
} 
dois termos ainda a serem apresentados: $\tau$, parâmetro da geodésica, é chamado parâmetro afim, e no caso de geodésicas percorridas por um corpo com massa ou um observador, $\tau$ é o tempo próprio marcado pelo relógio transportado pelo observador. Outro termo de (7) é $\Gamma_{\nu \gamma}^{\mu}$, chamado conexão afim, que é um tensor calculado a partir da métrica $g_{\mu \nu}$, ou seja, é diferente para cada geometria ou espaço-tempo. A conexão afim é definida como

$$
\Gamma_{\nu \gamma}^{\mu}=\frac{1}{2} g^{\mu \rho}\left(\frac{\partial g_{\gamma \rho}}{\partial x^{\nu}}+\frac{\partial g_{\nu \rho}}{\partial x^{\gamma}}-\frac{\partial g_{\nu \gamma}}{\partial x^{\rho}}\right) .
$$

Para um espaço-tempo plano como o de Minkowski, a conexão afim é zero.

Carter, para construir as geodésicas de Kerr, partiu da equação de Hamilton-Jacobi. Determinou que um corpo ou uma partícula de teste ao longo de uma geodésica no espaço-tempo (2) tem quatro constantes de movimento. A primeira é a massa $m$ da partícula de teste, duas outras quantidades surgem diretamente pelas simetrias da métrica de Kerr. Tal métrica não possui termos que dependam das coordenadas $t$ e $\phi$, logo, pelos manuais de geometria diferencial, a métrica de Kerr tem quantidades conservadas relacionadas à independência de tais coordenadas. A independência em relação à $t$ e $\phi$ diz-nos que a métrica de Kerr tem dois campos vetoriais importantes, dois campos de Killing, $\xi_{t}$ e $\xi_{\phi}$, e tais campos estão relacionados a duas constantes no movimento da partícula ao longo da geodésica - são elas $E$ and $L$, i.e., a energia (dada pela componente temporal do momento generalizado) e o momento angular (dado pela componente azimutal do momento generalizado):

$$
p_{t}=-E \quad \text { e } \quad p_{\phi}=L .
$$

Por fim, a última quantidade conservada é hoje conhecida como constante de Carter, que, como veremos, será indicada pela letra $K$. A constante de Carter é a constante de separação das equações da geodésica.

O método de Carter mostrou a separabilidade das equações da geodésica, conduzindo-nos a equações independentes para uma geodésica e à sua constante $K$. A partir da equação de Hamilton-Jacobi

$$
\frac{\partial S}{\partial \tau}=-\frac{1}{2} g^{\mu \nu} \frac{\partial S}{\partial x^{\mu}} \frac{\partial S}{\partial x^{\nu}}
$$

onde a função $S$ é a ação de Jacobi, que se relaciona ao momento generalizado pela seguinte equação

$$
p_{\mu} \equiv \frac{\partial S}{\partial x^{\mu}}
$$

Carter assumiu que $S$ pode ser escrita como

$$
S=\frac{1}{2} m^{2} \tau-E t+L \phi+S_{\theta}(\theta)+S_{r}(r) .
$$

As expressões para $S_{\theta}$ e $S_{r}$ podem ser omitidas por uma questão de economia de espaço ${ }^{13}$ Mesmo na teoria da relatividade geral, a partir da lagrangiana $\mathcal{L}$, o

\footnotetext{
13 Veja Neves [32], capítulo 4, para encontrar tais expressões e todo o caminho detalhado para a construção das geodésicas nos espaçostempo de Kerr, Kerr-de Sitter e Kerr-anti-de Sitter.
}

momento generalizado de uma partícula é definido por $p_{\mu} \equiv \frac{\partial \mathcal{L}}{\partial \dot{x}^{\mu}}=g_{\mu \nu} \dot{x}^{\nu}$, em que o operador ponto significa derivação em relação ao parâmetro afim, isto é, $d / d \tau$. Portanto, se usarmos essa definição de momento generalizado e a equação (12), substituindo-as na equação (11), teremos as equações das geodésicas. E para a métrica (2), tais equações (uma para cada componente na base das coordenadas $t, r, \theta$ e $\phi$ ) são melhor escritas e utilizadas como derivadas em relação ao parâmetro afim, ou seja,

$$
\begin{aligned}
\frac{d t}{d \tau} & =\frac{1}{\Sigma}\left[\frac{\left(r^{2}+a^{2}\right) P}{\Delta}-a\left(a E \operatorname{sen}^{2} \theta-L\right)\right] \\
\frac{d r}{d \tau} & =\frac{\sqrt{\mathcal{R}}}{\Sigma} \\
\frac{d \theta}{d \tau} & =\frac{\sqrt{\Theta}}{\Sigma} \\
\frac{d \phi}{d \tau} & =\frac{1}{\Sigma}\left[\frac{a P}{\Delta}-\left(a E-\operatorname{cossec}^{2} \theta L\right)\right] .
\end{aligned}
$$

As funções $P, \mathcal{R}$ e $\Theta$ são definidas como

$$
\begin{aligned}
P & =\left(r^{2}+a^{2}\right) E-a L \\
\mathcal{R} & =P^{2}-\Delta\left(m^{2} r^{2}+K\right) \\
\Theta & =Q-\cos ^{2} \theta\left[a^{2}\left(m^{2}-E^{2}\right)+\operatorname{cossec}^{2} \theta L^{2}\right]
\end{aligned}
$$

As quatro equações (13)- 16 fornecem as componentes de uma geodésica percorrida por uma partícula de massa $m$ na geometria ou espaço-tempo de Kerr, cuja métrica é dada pela equação 2 . Em particular para o fenômeno sombra, o parâmetro $m$, a massa da partícula, é nulo, pois estamos lidando com fótons ou partículas de luz e raios de luz que nos permitem traçar a silhueta da sombra. Por fim, a última constante ao longo de uma geodésica no espaçotempo de Kerr, a constante de Carter, é relacionada à constante $Q$, na equação 19 , por

$$
Q=K-(L-a E)^{2} \text {. }
$$

Vale a pena notar que $Q$ é nula para órbitas equatoriais, pois $d \theta / d \tau=\Theta=0$, ou seja, para órbitas equatoriais $\theta=\pi / 2$ conduz à $Q=0$. E a constante de Carter pode então ser apresentada como $K=(L-a E)^{2}$, ou seja, como uma constante a partir de constantes de movimento da partícula e do parâmetro de rotação do buraco negro.

\subsection{A silhueta da sombra}

Com as equações das geodésicas disponíveis, as quatro equações (13)- 16 , estamos aptos a obter as órbitas que delimitam a silhueta da sombra do buraco negro de Kerr. A silhueta é dada por órbitas instáveis $r=r_{f}$, fora do horizonte de eventos, tais que fótons em tais órbitas possam cair no buraco negro ou escapar até o observador. Para obter tais órbitas, equações fornecidas pela componente radial das geodésicas são resolvidas. As condições para a obtenção das órbitas instáveis são

$$
\mathcal{R}\left(r_{f}\right)=0 \quad \text { e }\left.\quad \frac{d}{d r} \mathcal{R}(r)\right|_{r=r_{f}}=0 .
$$


Para um buraco negro com rotação como o de Kerr, temos os valores $r_{f-}$ e $r_{f+}$ (sendo que $r_{f-} \leq r_{f+}$ ), i.e., existem um valor mínimo e um valor máximo para o raio das órbitas instáveis $r_{f}$. Como disse, a silhueta da sombra, seu contorno, será dado pelos valores que $r_{f}$ assume. Como veremos, os lados direito e esquerdo da sombra não são iguais para quaisquer conjuntos de parâmetros de um buraco negro em rotação. Isso deve-se ao efeito do arrasto do espaço-tempo, algo ausente em buracos negros sem rotação como o de Schwarzschild. Para a geometria de Schwarzschild, $r_{f-}=r_{f+}=3 M$, e a sombra é um círculo perfeito. Já para Kerr, $r_{f-}$ e $r_{f+}$ não são necessariamente iguais, e a sombra pode exibir uma deformação, que no caso de M87*, como disse, é um desvio da circularidade menor do que $10 \%$.

Na silhueta da sombra, costuma-se definir duas constantes úteis, dois parâmetros de impacto, que evolvem parâmetros das partículas ou fótons ao longo das geodésicas. São elas

$$
\xi=\frac{L}{E} \quad \text { e } \quad \eta=\frac{K}{E^{2}}
$$

As condições indicadas na equação (21) serão usadas e permitem-nos escrever $\xi$ e $\eta$ como dependentes do raio $r_{f}$, que delimita a silhueta da sombra. Ou seja, agora temos

$$
\eta\left(r_{f}\right)=\left.\frac{16 r^{2} \Delta}{\Delta^{\prime 2}}\right|_{r=r_{f}}
$$

$$
\xi\left(r_{f}\right)=\left.\frac{\left(r^{2}+a^{2}\right) \Delta^{\prime}-4 r \Delta}{a \Delta^{\prime}}\right|_{r=r_{f}},
$$

com o símbolo ' indicando derivada em relação à coordenada radial, isto é, $d / d r$. Para podermos mapear a sombra e usarmos as constantes definidas acima, costumase adotar uma base (uma tetrada) definida na posição do observador de tal forma que os raios de luz, que percorrem uma geodésica nula, possam ser decompostos em componentes com o auxílio da nova base (veja o Apêndice A para encontrar a tetrada aqui adotada). Ou seja, raios de luz cujas curvas são indicadas por $\gamma$ são escritos em termos da nova base ortonormal $\left(\mathbf{e}_{\mathbf{0}}, \mathbf{e}_{\mathbf{1}}, \mathbf{e}_{\mathbf{2}}, \mathbf{e}_{\mathbf{3}}\right)$. Raios que passam pela região $r_{f-} \leq r \leq r_{f+}$ são escritos a partir da tetrada e dos ângulos celestes $\alpha$ e $\beta$ (veja figura 4). Tais raios são especificados pelos parâmetros $\xi\left(r_{f}\right)$ e $\eta\left(r_{f}\right)$, que são constantes ao longo da geodésica, tornando as geodésicas que passam pela silhueta da sombra identificáveis.

Depois de muita álgebra (com o caminho às coordenadas celestes indicado no Apêndice A, as coordenadas que descrevem a silhueta da sombra do buraco negro de Kerr ficam

$$
\begin{aligned}
\operatorname{sen} \alpha & =\left.\frac{\sqrt{\Delta \eta\left(r_{f}\right)}}{\left(r^{2}+a^{2}\right)-a \xi\left(r_{f}\right)}\right|_{\left(r_{o b s}, \theta_{o}\right)}, \\
\operatorname{sen} \beta & =\left.\frac{\left(\xi\left(r_{f}\right) \operatorname{cossec}^{2} \theta-a\right) \operatorname{sen} \theta}{\sqrt{\eta\left(r_{f}\right)}}\right|_{\left(r_{o b s}, \theta_{o}\right)} .
\end{aligned}
$$

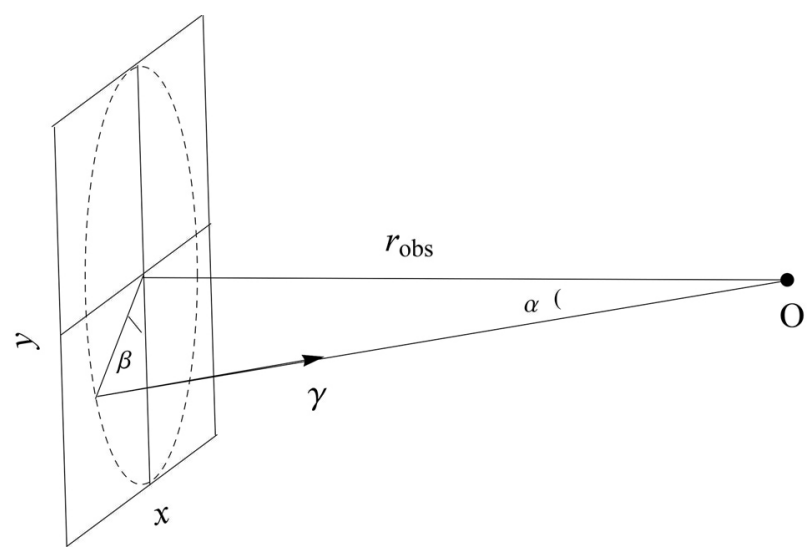

Figura 4: As coordenadas celestes, os ângulos $\alpha$ e $\beta$, descrevem a sombra do buraco negro. A silhueta da sombra, linha tracejada, é descrita pelo observador $O$ a partir de raios de luz (ou geodésicas do tipo luz) indicados por $\gamma$ que dão o contorno da sombra. A projeção estereográfica faz com que a sombra seja desenhada a partir das coordenadas cartesianas $x$ e $y$.

Como podemos diretamente ler, as coordenadas celestes dependem de $r_{o b s}$ e $\theta_{o}$, parâmetros que definem a posição do observador, e dos parâmetros das órbitas dos fótons na silhueta da sombra. E, claro, as coordenadas celestes dependem também dos parâmetros do buraco negro pois são funções de termos da métrica (2).

Para uma projeção estereográfica da sombra no plano, assume-se que o observador está bem distante do buraco negro, i.e., $r_{o b s}$ é grande, de tal forma que as coordenadas cartesianas são escritas como

$$
\begin{aligned}
& x\left(r_{f}\right)=-2 \operatorname{tg}\left(\frac{\alpha}{2}\right) \operatorname{sen} \beta, \\
& y\left(r_{f}\right)=-2 \operatorname{tg}\left(\frac{\alpha}{2}\right) \cos \beta .
\end{aligned}
$$

Ou seja, de acordo com a figura $4 x\left(r_{f}\right)$ e $y\left(r_{f}\right)$ são frutos da projeção das coordenadas (ou ângulos) celestes $\alpha$ e $\beta$ no plano cartesiano e formam um conjunto de equações paramétricas que desenham a silhueta da sombra.

Como podemos notar a partir da figura 5, que nos indica sombras para o buraco negro de Kerr para alguns parâmetros diferentes, o fenômeno é simétrico em relação ao eixo $x$, ou seja, a sombra assume os mesmos valores no eixo $y$ em ambos lados, o positivo e o negativo. Para um buraco negro como o de Kerr, a imagem da sombra depende fortemente do parâmetro de rotação ou do parâmetro de rotação adimensional, indicado por $a_{*}=a / M$, e do ângulo em que é observada, $\theta_{o}$. Quanto mais rotação, mais deslocada à direita, em relação ao eixo $y$, a sombra fica. Vale a pena destacar a deformação da sombra, algo que, como já foi dito, somente é possível para um buraco negro em rotação. A rotação do buraco provê o arrasto do espaço-tempo, torna impossível algo estático na ergosfera, i.e., a rotação do buraco negro carrega tudo, inclusive fótons que orbitam naquela região. É o arrasto do espaço-tempo a causa da deformação da sombra. Nas sombras da figura 5 a rotação do buraco negro é da 

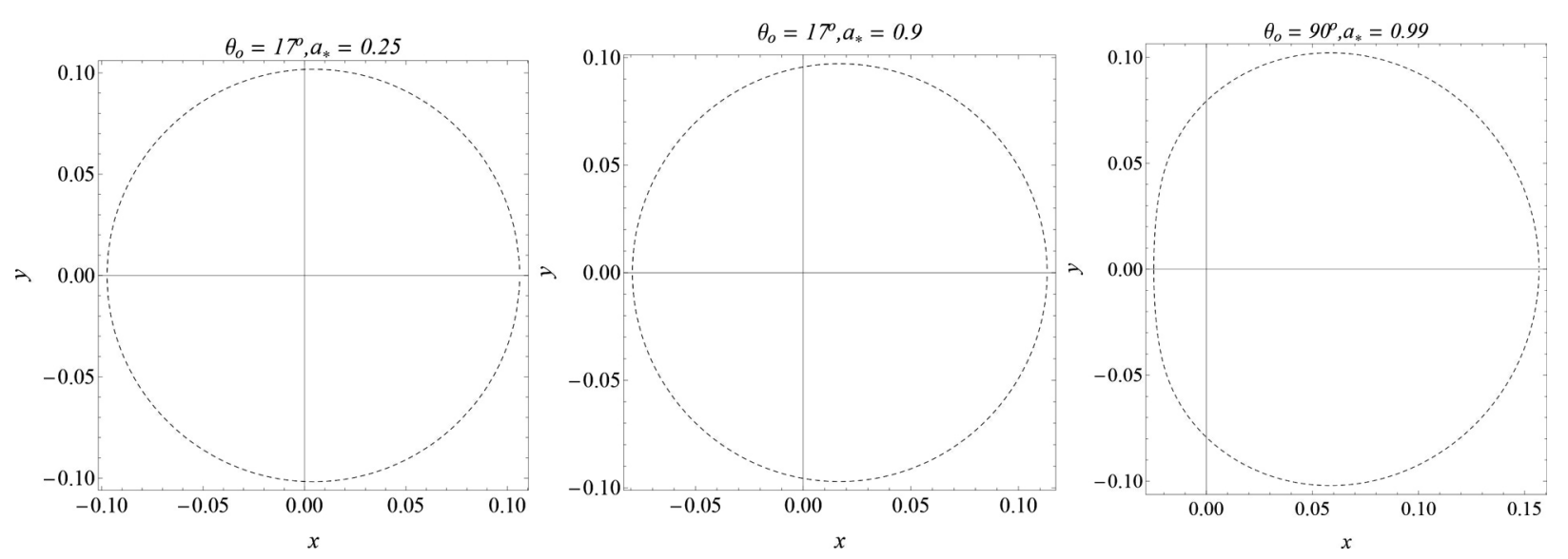

Figura 5: Silhuetas de sombras produzidas por um buraco negro de Kerr. Escritas a partir das coordenadas $x$ e $y$, podemos notar que a sombra deforma-se mais para altos valores do ângulo $\theta_{o}$ em que é observada e do parâmetro de rotação adimensional $a_{*}=a / M$. Há fortes indícios no sentido de que a sombra de M87* foi observada num ângulo $\theta_{o}=17^{\circ}$. Outra característica na projeção da sombra é o seu deslocamento para direita e a sua deformação à esquerda, produzida pela rotação do buraco negro. Aqui é assumida a rotação no sentido anti-horário, ou seja, à esquerda fótons viajam no mesmo sentido da rotação do buraco, já à direita, a viagem é contra a rotação. Nas sombras esboçadas, utilizei as unidades geométricas $(G=c=1)$ e assumi $M=1$ e $r_{o b s}=50 M$ por conveniência.

esquerda para a direita. Como os raios de luz vêm por trás do buraco negro (a partir do fundo estrelado), à esquerda na sombra temos as trajetórias dos raios que acompanham a rotação do buraco negro, já à direita temos um movimento no sentido inverso da rotação. À esquerda as trajetórias são dadas por $r_{f-}$, são trajetórias que avançam. À direita as trajetórias são dadas por $r_{f+}$, são as trajetórias retrógradas. Essa diferença entre as trajetórias provoca o achatamento da sombra no seu lado esquerdo, algo que fica mais pronunciado para altos valores do parâmetro de rotação e para grandes ângulos de observação, cuja deformação máxima está em $\theta_{o}=90^{\circ}$.

$\mathrm{Na}$ imagem obtida pela colaboração, como disse, te$\operatorname{mos} \theta_{o}=17^{\circ}$, e a deformação da sombra de M87* neste ângulo exclui pequenos valores do parâmetro de rotação, ou seja, $a_{*} \simeq 0$ é praticamente descartado. Estimativas posteriormente publicadas à imagem, feitas a partir da matéria atraída por M87*, afirmam um valor mínimo para o parâmetro de rotação, $a_{*}>0.4$ em Nemmen 33], e até mesmo um valor com boa precisão, $a_{*}=0.90 \pm 0.05$ em Tamburini et al. [34]. No último trabalho citado, é afirmado, com um grandíssimo nível de confiança estatística, que M87* está rodando. Parâmetros de rotação serão ainda melhor determinados em futuras sombras. Com mais sombras no futuro, tratamentos estatísticos poderão ser realizados para cada parâmetro de uma sombra e do buraco negro que a gera, melhorando ainda mais os valores até agora estimados.

Por fim, com a melhor estimativa do parâmetro de rotação de M87* discutida acima (além do preciso valor para a sua massa obtido pela EHT), pode-se calcular o valor do horizonte de eventos de M87* a partir da equação (5). Calculando-o, temos $r_{h} \simeq 1.4 \times 10^{10} \mathrm{~km}$ ou um raio de um horizonte de eventos maior do que o raio da órbita de Plutão! Já os raios das órbitas que formam a silhueta da sombra ficam entre $2.1 \times 10^{10} \mathrm{~km} \lesssim r_{f} \lesssim 2.9 \times 10^{10} \mathrm{~km}$. Ou seja, a sombra tem um tamanho na escala do horizonte de eventos, possui um raio que é um pouco maior do que o dobro do raio do horizonte de eventos. Este é motivo pelo qual a colaboração afirmou, peremptoriamente, que foram construídas imagens de M $87^{*}$ na escala do seu horizonte de eventos.

\section{Aplicações das Sombras}

Acima de tudo, sombras de buracos negros são testes à teoria da relatividade geral quando campos gravitacionais fortíssimos estão envolvidos. Como vimos, por causa da imensa massa do girante M87* ser bilhões de vezes a massa solar, efeitos gravitacionais que são somente previstos pela teoria einsteiniana, como o arrasto do espaço-tempo, são então colocados a teste. Sombras colocam em teste modelos, geometrias, métricas geradas a partir das equações de Einstein. Não apenas, as sombras podem também colocar em teste teorias gravitacionais que estão além da teoria da relatividade geral, teorias que supostamente sucederiam a teoria einsteiniana em regimes de altas energias. Seleciono aqui alguns trabalhos atuais que usam parâmetros da sombra de M87* (e de futuras sombras) para obter vínculos e limites a parâmetros de teorias, modelos e geometrias:

- Uso da sombra e da matéria em acreção por M87* para a determinação da rotação do buraco negro [33, 34, inclusive para propor, como em Bambi et al. 35, um superspinar, um buraco negro em que $a^{2}>M^{2}$ ou $a_{*}>1$ como origem da famosa imagem. Propostas para alternativas ao buraco negro de Kerr na descrição dos parâmetros de M87* incluem buracos negros regulares 36 e buracos negros em 
eletrodinâmica não linear 37]. Como mais um teste da teoria da relatividade geral a partir de M87*, Yan et al. [38] estudaram uma possível violação do princípio de equivalência (um dos princípios fundacionais da teoria einsteiniana) num regime de intenso campo gravitacional.

- Tsupko et al. 39 sugerem o uso da sombra para obter parâmetros cosmológicos, como o parâmetro de Hubble. Uma sombra poderá ser, de acordo com os autores, uma régua padrão assim como uma supernova o é.

- Determinação de parâmetros de modelos e teorias, com o uso de sombras, em propostas que tentam ir além da teoria de Einstein. Parâmetros como o raio da dimensão extra em mundos brana [40] ou a influência dessa mesma dimensão extra em nosso universo quadridimensional, por meio de uma carga de maré 41 42, foram estimados. No trabalho [36], a sombra de M $87^{*}$ produziu um limite superior ao parâmetro de gravidade quântica de um princípio de incerteza generalizado, um princípio que torna o princípio de incerteza de Werner Heisenberg um caso particular. Já Liu et al. [20], à luz dos parâmetros de M87* estudaram a sombra de um buraco negro numa proposta de unificação da teoria da relatividade e da mecânica quântica, a gravidade quântica em loop (loop quantum gravity).

É praticamente certo que a construção de novas sombras de outros buracos negros supermassivos, seja a partir do trabalho da EHT ou de novas colaborações, equipamentos e técnicas 14 produzirá estatísticas a partir das quais parâmetros — seja em gravitação, cosmologia, teoria quântica de campos — serão melhor estimados, ou seja, com maior precisão. Sombras como a do buraco negro M87* serão mais uma forma para investigar fenômenos e estabelecer limites mais precisos, acima de tudo, quando campos gravitacionais intensos estiverem em jogo.

\section{Comentários Finais}

A sombra do buraco negro M87*, no centro da galáxia Messier 87, foi a mais comentada e divulgada imagem científica no ano de 2019. A sombra produzida por um buraco negro a milhões de anos-luz daqui e com uma massa de bilhões de vezes a massa do Sol despertou a curiosidade e o medo de muitos. A colaboração internacional que construiu tal feito, a Event Horizon Telescope, não hesitou em afirmar que a teoria da relatividade geral de Albert Einstein foi aprovada no novo teste, e a sombra de M87* pôde ser bem descrita por uma das mais estudadas soluções das equações do campo gravitacional

\footnotetext{
${ }^{14}$ Equipamentos como o futuro telescópio de 30 metros 43, no Havaí, e a rede de nova geração de radiotelescópios NGVLA (Next Generation Very Large Array) 44 .
}

ou equações de Einstein: a solução ou buraco negro de Roy Kerr.

O buraco negro de Kerr é um buraco negro em rotação. Por estar em rotação, pode produzir sombras deformadas a partir de conjuntos específicos de parâmetros, em conformidade à sombra relatada pela colaboração internacional. Neste trabalho, a solução de Kerr foi apresentada, e suas principais características foram destacadas. Um procedimento para o cálculo de sombras foi introduzido. Com isso, fomos capazes de enxergar o efeito da rotação na sombra. Fomos capazes de confirmar a colaboração.

Por fim, elenquei algumas aplicações das sombras (a atual de M87* e as vindouras) na gravitação e na cosmologia. O estudo de sombras apresenta-se, acima de tudo, como um estudo a partir de campos gravitacionais intensos, gerados por buracos negros com milhões e bilhões de massas solares. Em tal contexto, diferente de testes e experimentos feitos no sistema solar, modelos, teorias, teoremas e conjecturas poderão ser melhor determinados e testados. O "fim do mundo" indicado por uma sombra poderá ser o começo de um novo mundo dentro da física.

\section{Agradecimentos}

Agradeço à Coordenação de Aperfeiçoamento de Pessoal de Nível Superior (CAPES) o apoio financeiro.

\section{Apêndice}

\section{A. Construção das coordenadas celestes}

As coordenadas celestes são adotadas para que se desenhe a silhueta de uma sombra. E um distante observador a desenhará a partir do seu referencial ou da sua tetrada. Uma tetrada $\left(\mathbf{e}_{\mathbf{0}}, \mathbf{e}_{\mathbf{1}}, \mathbf{e}_{\mathbf{2}}, \mathbf{e}_{\mathbf{3}}\right)$ pode ser vista como uma base a partir da qual vetores e tensores são escritos. Então, ao invés da base das coordenadas $t, r, \theta$ e $\phi$, é usada uma tetrada. E numa geometria como a de Kerr, o formalismo das tetradas pode simplificar muitas equações $\sqrt{15}$ De acordo com Grenzebach et al. [14], a tetrada ortonormal escolhida - a partir da qual projetam-se os raios de luz ou as geodésicas tipo luz — é escrita como

$$
\begin{aligned}
\mathbf{e}_{\mathbf{0}} & =\left.\frac{1}{\sqrt{\Delta \Sigma}}\left[\left(r^{2}+a^{2}\right) \frac{\partial}{\partial t}+a \frac{\partial}{\partial \phi}\right]\right|_{\left(r_{o b s}, \theta_{o}\right)}, \\
\mathbf{e}_{\mathbf{1}} & =\left.\sqrt{\frac{1}{\Sigma}} \frac{\partial}{\partial \theta}\right|_{\left(r_{o b s}, \theta_{o}\right)}, \\
\mathbf{e}_{\mathbf{2}} & =-\left.\frac{1}{\sqrt{\Sigma} \operatorname{sen} \theta}\left(\frac{\partial}{\partial \phi}+a \operatorname{sen}^{2} \theta \frac{\partial}{\partial t}\right)\right|_{\left(r_{o b s}, \theta_{o}\right)}, \\
\mathbf{e}_{\mathbf{3}} & =-\left.\sqrt{\frac{\Delta}{\Sigma}} \frac{\partial}{\partial r}\right|_{\left(r_{o b s}, \theta_{o}\right)} .
\end{aligned}
$$

\footnotetext{
${ }^{15}$ Veja Carroll [26], apêndice $\mathrm{J}$, para uma discussão sobre o formalismo das tetradas.
} 
A base $\left(\mathbf{e}_{\mathbf{0}}, \mathbf{e}_{\mathbf{1}}, \mathbf{e}_{\mathbf{2}}, \mathbf{e}_{\mathbf{3}}\right)$, calculada na posição do observador $\left(r_{o b s}, \theta_{o}\right)$, é escrita em componentes dos vetores da base das coordenadas $\left(\frac{\partial}{\partial t}, \frac{\partial}{\partial r}, \frac{\partial}{\partial \theta}, \frac{\partial}{\partial \phi}\right)$. As características da tetrada acima são: $\mathbf{e}_{\mathbf{0}}$ é a quadrivelocidade do observador, e $\mathbf{e}_{3}$ aponta na direção do buraco negro, ou seja, na direção $\frac{\partial}{\partial r}$. Além disso, é escolhida a direção $\mathbf{e}_{\mathbf{0}} \pm \mathbf{e}_{\mathbf{3}}$ como tangencial à direção das congruências nulas principais, que são usadas para caracterizar espaços-tempo na teoria da relatividade geral ${ }^{16}$ Com a tetrada acima, o observador não está necessariamente em repouso, além de não estar no "infinito". É comum em trabalhos que tratam de espaços-tempo assintoticamente planos assumir que $r_{o b s} \rightarrow \infty$ para a descrição da sombra. No entanto, o caminho adotado aqui permite observadores distantes mas não obrigatoriamente no "infinito". Com isso, a abordagem exposta neste apêndice permite o estudo de sombras de buracos negros em espaços-tempos que não são assintoticamente planos, ou seja, que não são o espaço-tempo de Minkowski quando $r_{o b s} \rightarrow \infty$. Exemplos de tais espaços-tempo são os assintoticamente de Sitter e anti-de Sitter, como estudados nas referências $14,19,36$.

Para descrever os raios de luz que passam por $r_{f}$ (a região de fótons que marca a silhueta da sombra) e chegam ao observador, as curvas que os representam, ou seja, as curvas $\gamma$ cujos vetores tangentes são do tipo luz, têm vetores tangentes na base das coordenadas dados por

$$
\frac{d \gamma}{d \tau}=\left(\frac{d t}{d \tau}\right) \frac{\partial}{\partial t}+\left(\frac{d r}{d \tau}\right) \frac{\partial}{\partial r}+\left(\frac{d \theta}{d \tau}\right) \frac{\partial}{\partial \theta}+\left(\frac{d \phi}{d \tau}\right) \frac{\partial}{\partial \phi} .
$$

Como disse, precisamos de uma descrição do fenômeno na posição em que se encontra o observador, portanto o vetor acima será decomposto em termos da tetrada. Decompondo-o, fica

$$
\frac{d \gamma}{d \tau}=\zeta\left(-\mathbf{e}_{0}+\operatorname{sen} \alpha \cos \beta \mathbf{e}_{1}+\operatorname{sen} \alpha \operatorname{sen} \beta \mathbf{e}_{2}+\cos \alpha \mathbf{e}_{3}\right),
$$

(34)

de tal forma que os ângulos $\alpha$ and $\beta$ são as já indicadas coordenadas celestes (caracterizadas na figura 4). Como afirmado na subseção 3.3 , a descrição da sombra é feita pelas coordenadas celestes, depois de projetadas estereograficamente no plano cartesiano.

Com a decomposição (34) do vetor tangente aos raios de luz com o uso da tetrada $\left(\mathbf{e}_{\mathbf{0}}, \mathbf{e}_{1}, \mathbf{e}_{2}, \mathbf{e}_{3}\right)$, o fator $\zeta$ pôde ser escrito como

$$
\zeta=-\left.\frac{\left(r^{2}+a^{2}\right) E-a L}{\sqrt{\Delta \Sigma}}\right|_{\left(r_{o b s}, \theta_{o}\right)} .
$$

\footnotetext{
${ }^{16}$ Existem formas de classificar soluções do campo gravitacional na relatividade geral, como a solução de Kerr, a partir do tensor de curvatura ou do tensor de Weyl e suas propriedades. Na classificação que usa o tensor de curvatura e direções principais nulas, atribuída a Aleksei Petrov, a solução de Kerr é tida como algebricamente especial, tem até dois vetores nulos principais diferentes. Veja Wald [31], capítulo 7, para um estudo sobre as classificações de soluções e, em especial, os dois vetores nulos principais da geometria de Kerr encontram-se na p. 313.
}

E, por fim, as coordenadas celestes foram igualmente determinadas na configuração e geometria adotadas:

$$
\begin{aligned}
\cos \alpha & =\left.\frac{\Sigma \frac{d r}{d \tau}}{\left(r^{2}+a^{2}\right) E-a L}\right|_{\left(r_{o b s}, \theta_{o}\right)}, \\
\operatorname{sen} \beta & =\left.\frac{\operatorname{sen} \theta}{\sqrt{\Delta} \operatorname{sen} \alpha}\left(\frac{\Delta \Sigma \frac{d \phi}{d \tau}}{\left(r^{2}+a^{2}\right) E-a L}-a\right)\right|_{\left(r_{o b s}, \theta_{o}\right)}
\end{aligned}
$$

Portanto, se usarmos as equações para as componentes $r$ e $\phi$ da geodésica, indicadas nas equações (14) e (16), e as substituirmos nas equações (36)-(37), obteremos as formas para $\alpha$ e $\beta$ (ou $\operatorname{sen} \alpha$ e $\operatorname{sen} \beta$ ) mostradas na subseção 3.3

\section{Referências}

[1] K. Akiyama, A. Alberdi, R. Azulay, A.K. Baczko, M. Baloković, J. Barrett, L. Blackburn, K.L. Bouman, G.C. Bower, C.D. Brinkerink et al., Astrophys. J. Lett. 875, L1 (2019).

[2] K. Akiyama, A. Alberdi, R. Azulay, A.K. Baczko, M. Baloković, J. Barrett, L. Blackburn, K.L. Bouman, G.C. Bower, C.D. Brinkerink et al., Astrophys. J. Lett. 875, L6 (2019).

[3] G.E.A. Matsas, Rev. Bras. Ensino Fis. 27, 137 (2005).

[4] D. Alighieri, A Divina Comédia: Inferno (Editora 34, São Paulo, 2010).

[5] A. Einstein, Sitzungsber. K. Preuß. Akad. Wiss. 1, 688 (1916)

[6] R.P. Kerr, Phys. Rev. Lett. 11, 26 (1963).

[7] K. Schwarzschild, Sitzungsber. K. Preuß. Akad. Wiss. 1916, 189 (1916).

[8] A. Saa, Rev. Bras. Ensino Fis. 38, e4201 (2016).

[9] J.L. Synge, Mon. Not. R. Astron. Soc. 131, 463 (1966).

[10] J.M. Bardeen, Timelike and Null Geodesics in the Kerr Metric, in Black Holes, edited by C. DeWitt and B. DeWitt (Gordon and Breach, New York, 1973), p. 215.

[11] A.F. Zakharov, F. De Paolis, G. Ingrosso, A.A. Nucita, Astron. Astrophys. 442, 795 (2005).

[12] A. de Vries, Classical Quantum Gravity 17, 123 (2000).

[13] V. Perlick, O.Y. Tsupko, G.S. Bisnovatyi-Kogan, Phys. Rev. D. 97, 104062 (2018).

[14] A. Grenzebach, V. Perlick, C. Lämmerzahl, Phys. Rev. D. 89, 124004 (2014).

[15] Z. Li, C. Bambi, J. Cosmol. Astropart. Phys. 01, 041 (2014).

[16] A. Abdujabbarov, M. Amir, B. Ahmedov, S.G. Ghosh, Phys. Rev. D. 93, 104004 (2016).

[17] M. Amir, S.G. Ghosh, Phys. Rev. D. 94, 024054 (2016).

[18] L. Amarilla, E.F. Eiroa, Phys. Rev. D. 85, 064019 (2012).

[19] E.F. Eiroa, C.M. Sendra, Eur. Phys. J. C. 78, 91 (2018).

[20] C. Liu, T. Zhu, Q. Wu, K. Jusufi, M. Jamil, M. AzregAïnou, A. Wang, Phys. Rev. D. 101, 084001 (2020).

[21] J.A.S. Lima, R.C. Santos, Rev. Bras. Ensino Fis. 41, e20190199 (2019).

[22] J.P.S. Lemos, Rev. Bras. Ensino Fis. 41, e20190260 (2019).

[23] H.L. César, P.J. Pompeia, N. Studart, Rev. Bras. Ensino Fis. 41, e20190238 (2019). 
[24] P.V.P. Cunha, C.A.R. Herdeiro, Gen. Rel. Grav. 50, 42 (2018).

[25] R.H. Boyer, R.W. Lindquist, J. Math. Phys. 8, 265 (1967).

[26] S. Carroll, Spacetime and Geometry: an Introduction to General Relativity (Addison Wesley, San Francisco, 2004).

[27] J. Neves, Rev. Bras. Ensino Fis. 39, e3303 (2017).

[28] J.C.S. Neves, Found. Sci. 25, 857 (2020).

[29] R. Penrose, Riv. del Nuovo Cim. 1, 252 (1969). Republicado em Gen. Rel. Grav. 34, 1141 (2002).

[30] B. Carter, Phys. Rev. 174, 1559 (1968).

[31] R.M. Wald, General Relativity (The University of Chicago Press, Chicago, 1984)

[32] J.C.S. Neves, Mundos Brana: Buracos Negros e Buracos de Minhoca. Tese de Doutorado, Universidade de São Paulo, São Paulo, 2012.

[33] R. Nemmen, Astrophys. J. Lett. 880, L26 (2019).

[34] F. Tamburini, B. Thidé, M.D. Valle, Mon. Not. R. Astron. Soc. 492, L22 (2020).

[35] C. Bambi, K. Freese, S. Vagnozzi, L. Visinelli, Phys. Rev. D. 100, 044057 (2019).

[36] J.C.S. Neves, Eur. Phys. J. C. 80, 343 (2020).

[37] A. Allahyari, M. Khodadi, S. Vagnozzi, D.F. Mota, JCAP 02, 003 (2020).

[38] S.F. Yan, C.Li, L. Xue, X. Ren, Y.F. Cai, D.A. Easson, Y.F. Yuan, H. Zhao, Phys. Rev. Research 2, 023164 (2020).

[39] O.Y. Tsupko, Z. Fan, G.S. Bisnovatyi-Kogan, Class. Quant. Grav. 37, 065016 (2020).

[40] S. Vagnozzi, L. Visinelli, Phys. Rev. D. 100, 024020 (2019).

[41] J.C.S. Neves, Eur. Phys. J. C. 80, 717 (2020).

[42] I. Banerjee, S. Chakraborty, S. SenGupta, Phys. Rev. D. 101, 041301 (2020).

[43] G.H. Sanders, J. Astrophys. Astron. 34, 81 (2013).

[44] A.M. Hughes, A. Beasley, C. Carilli, IAU General Assembly 22, 2255106 (2015). 